\title{
Evolution of newly formed dust in Population III supernova remnants and its impact on the elemental composition of Population II.5 stars
}

\author{
Takaya Nozawa ${ }^{1,2}$, Takashi Kozasa ${ }^{1}$, Asao Habe ${ }^{1}$, Eli Dwek ${ }^{3}$, \\ Hideyuki Umeda ${ }^{4}$, Nozomu Tominaga ${ }^{5}$, Keiichi Maeda ${ }^{2,6}$, and \\ Ken'ichi Nomoto ${ }^{2,4,7}$ \\ ${ }^{1}$ Department of Cosmosciences, Graduate School of Science, Hokkaido University, \\ Sapporo 060-0810, Japan \\ email: tnozawa@mail.sci.hokudai.ac.jp \\ ${ }^{2}$ Institute for the Physics and Mathematics of the Universe, University of Tokyo, \\ Kashiwa, Chiba 277-8568, Japan \\ ${ }^{3}$ Laboratory for Astronomy and Solar Physics, NASA Goddard Space Flight Center, \\ Greenbelt, MD 20771, USA \\ ${ }^{4}$ Department of Astronomy, School of Science, University of Tokyo, \\ Bunkyo-ku, Tokyo 113-0033, Japan \\ ${ }^{5}$ Division of Optical and Infrared Astronomy, National Astronomical Observatory of Japan, \\ Mitaka, Tokyo 181-8588, Japan \\ ${ }^{6}$ Max-Planck-Institut für Astrophysik, 85741 Garching, Germany \\ ${ }^{7}$ Research Center for the Early Universe, School of Science, University of Tokyo, \\ Bunkyo-ku, Tokyo 113-0033, Japan
}

\begin{abstract}
We investigate the evolution of dust formed in Population III supernovae (SNe) by considering its transport and processing by sputtering within the SN remnants (SNRs). We find that the fate of dust grains within SNRs heavily depends on their initial radii $a_{\text {ini }}$. For Type II SNRs expanding into the ambient medium with density of $n_{\mathrm{H}, 0}=1 \mathrm{~cm}^{-3}$, grains of $a_{\mathrm{ini}}<0.05 \mu \mathrm{m}$ are detained in the shocked hot gas and are completely destroyed, while grains of $a_{\text {ini }}>0.2 \mu \mathrm{m}$ are injected into the surrounding medium without being significantly destroyed. Grains with $a_{\text {ini }}=0.05-0.2 \mu \mathrm{m}$ are finally trapped in the dense shell behind the forward shock. We show that the grains piled up in the dense shell enrich the gas up to $10^{-6}-10^{-4} Z_{\odot}$, high enough to form low-mass stars with $0.1-1 M_{\odot}$. In addition, $[\mathrm{Fe} / \mathrm{H}]$ in the dense shell ranges from -6 to -4.5 , which is in good agreement with the ultra-metal-poor stars with $[\mathrm{Fe} / \mathrm{H}]<-4$. We suggest that newly formed dust in a Population III SN can have great impact on the stellar mass and elemental composition of Population II.5 stars formed in the shell of the SNR.
\end{abstract}

Keywords. dust, extinction, supernovae: general, hydrodynamics, shock waves, stars: abundances, stars: chemically peculiar, methods: numerical

\section{Introduction}

The first dust in the universe plays critical roles in the subsequent formation processes of stars and galaxies. Dust grains provide additional pathways for cooling of gas in metalpoor molecular clouds through their thermal emission and formation of $\mathrm{H}_{2}$ molecules on the surface (e.g., Cazaux \& Spaans 2004). In particular, the presence of dust decreases the value of the critical metallicity to $10^{-6}-10^{-4} Z_{\odot}$ (Omukai et al. 2005; Schneider et al. 2006; Tsuribe \& Omukai 2006), where the transition of star formation mode from massive Population III stars to low-mass Population II stars occurs. Since absorption and 
thermal emission by dust grains strongly depend on their composition, size distribution, and amount, it is essential to clarify the properties of dust in the early epoch of the universe, in order to elucidate the evolutionary history of stars and galaxies.

Dust grains at redshift $z>5$ are considered to have been predominantly produced in supernovae $(\mathrm{SNe})$. Theoretical studies have predicted that dust grains of $0.1-2 M_{\odot}$ and 10-60 $M_{\odot}$ are formed in the ejecta of primordial Type II SNe (SNe II, Todini \& Ferrara 2001; Nozawa et al. 2003) and pair-instability SNe (PISNe, Nozawa et al. 2003; Schneider et al. 2004), respectively. However, the newly formed dust is reprocessed via sputtering in the hot gas swept up by the reverse and forward shocks that are generated by the interaction between the SN ejecta and the surrounding medium. Thus, the size and mass of the dust can be greatly modified before being injected into the interstellar medium (ISM, Bianchi \& Schneider 2007; Nozawa et al. 2007).

Here we present the results of the calculations for the evolution of newly formed dust within Population III SN remnants (SNRs), based on the dust formation model by Nozawa et al. (2003). We investigate the transport of dust and its processing by sputtering in the shocked hot gas, and report the size and amount of dust injected from SNe into the ISM. It is also shown that a part of the surviving dust grains are piled up in the dense SN shell formed behind the forward shock and can enrich the gas in the dense shell up to $10^{-6}-10^{-4} Z_{\odot}$. We suppose that newly condensed dust in the SN ejecta has significant influence on the elemental abundances of Population II.5 stars, that is, the second-generation stars formed in the dense shell of Population III SNRs.

\section{Evolution of Dust in Population III SNRs}

We first briefly describe the models for the evolution of dust within SNRs. The time evolution of a SNR is numerically solved by assuming spherical symmetry. We adopt the hydrodynamic models of Population III SNe II with progenitor masses of $M_{\mathrm{pr}}=13$, 20, 25, and $30 M_{\odot}$ and explosion energy of $10^{51}$ ergs by Umeda \& Nomoto (2002) for the initial condition of the gas in the ejecta. For the ambient medium, we consider a uniform medium with a hydrogen number density of $n_{\mathrm{H}, 0}=0.1,1$, and $10 \mathrm{~cm}^{-3}$. For the model of dust in the He core, we adopt the dust grains formed in the unmixed ejecta by Nozawa et al. (2003). Treating dust grains as test particles, we calculate the destruction and dynamics of dust by taking into account the size distribution as well as the spatial mass distribution of each grain species.

The results of the calculations are shown in Figure 1. Figure $1 a$ shows the trajectories of $\mathrm{C}, \mathrm{Mg}_{2} \mathrm{SiO}_{4}$, and $\mathrm{Fe}$ grains within the SNR for $M_{\mathrm{pr}}=20 M_{\odot}$ and $n_{\mathrm{H}, 0}=1 \mathrm{~cm}^{-3}$, and Figure $1 b$ shows the time evolution of their radii relative to the initial ones. The positions of the forward shock, the reverse shock, and the surface of the He core are depicted by the thick solid lines in Figure 1a. Initially, newly formed dust grains are expanding with the cool gas in the ejecta, and thus they undergo neither gas drag nor processing by sputtering. However, once they intrude into the hot gas swept up by the reverse shock penetrating into the ejecta, they acquire the high velocities relative to the gas and are eroded by kinetic and/or thermal sputtering. The evolution of dust grains after colliding with the reverse shock heavily depends on their initial radii $a_{\text {ini }}$ and compositions.

For example, $\mathrm{C}$ grains formed in the outermost region of the He core encounter the reverse shock at $3650 \mathrm{yr}$. Since the deceleration by the gas drag is more efficient for smaller grains, $\mathrm{C}$ grains with $a_{\mathrm{ini}}=0.01 \mu \mathrm{m}$ quickly slow down. These small grains are eventually trapped in the hot gas $\left(\geqslant 10^{6} \mathrm{~K}\right)$ generated from the passage of the reverse and forward shocks and are completely destroyed by thermal sputtering. C grains of $a_{\text {ini }}=0.1$ $\mu \mathrm{m}$ reduce their sizes by sputtering but cannot be completely destroyed. These grains 


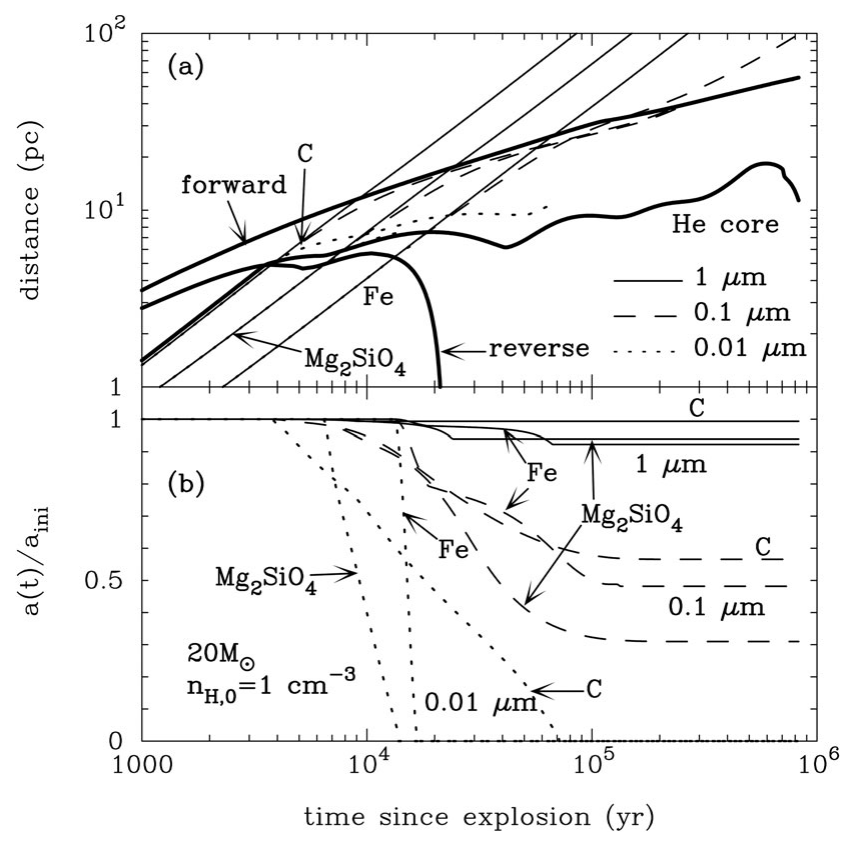

Figure 1. (a) Spatial evolution of $\mathrm{C}, \mathrm{Mg}_{2} \mathrm{SiO}_{4}$, and Fe grains within the SNR for $M_{\mathrm{pr}}=20 M_{\odot}$ and $n_{\mathrm{H}, 0}=1 \mathrm{~cm}^{-3}$ and $(b)$ the temporal evolution of their radii relative to the initial values. The evolution of dust with $a_{\mathrm{ini}}=0.01,0.1$, and $1 \mu \mathrm{m}$ is shown by the dotted, dashed, and solid lines, respectively. The thick solid lines in $(a)$ indicate the positions of the forward shock, the reverse shock, and the surface of the He core.

are finally captured in the dense SN shell formed behind the forward shock at $\sim 2 \times 10^{5}$ yr, where the gas temperature is too low $\left(<10^{5} \mathrm{~K}\right)$ to erode the dust grains by thermal sputtering. C grains with $a_{\mathrm{ini}}=1 \mu \mathrm{m}$, which are not efficiently decelerated by the gas drag, can pass through the forward shock front and are injected into the ambient medium without being significantly destroyed.

$\mathrm{Mg}_{2} \mathrm{SiO}_{4}$ grains, which are formed in the O-rich layer, collide with the reverse shock at about $6000 \mathrm{yr}$, but the dependence of their evolution on the initial radius is the same as for $\mathrm{C}$ grains. On the other hand, Fe grains formed in the innermost region of the ejecta hit the reverse shock after $13000 \mathrm{yr}$, and its $0.1 \mu \mathrm{m}$-sized grains are injected into the ambient medium because of the high bulk density.

As shown above, the small dust grains formed in the SN ejecta are predominantly destroyed by sputtering within the SNR. Thus, the size distribution of the surviving dust is dominated by larger grains, compared to the sizes at formation. Note that the evolution of dust within SNRs and thus the resulting size distribution of dust does not depend on the progenitor mass considered here, because their explosion energies are the same and the time evolution of the gas temperature and density within SNRs are similar. On the other hand, the ambient gas density strongly affects the evolution of dust in SNRs. The higher density in the ambient medium results in the higher density of the shocked gas and causes the efficient erosion and deceleration of dust due to more frequent collisions with the hot gas. Therefore, the initial radius below which dust is completely destroyed increases with increasing the ambient gas density and is 0.01, 0.05, and $0.2 \mu \mathrm{m}$ for $n_{\mathrm{H}, 0}=0.1,1$, and $10 \mathrm{~cm}^{-3}$, respectively. As a result, the total mass of the surviving dust is smaller for the higher ambient density and ranges from 0.01 to $0.8 M_{\odot}$ for $n_{\mathrm{H}, 0}=10$ to $0.1 \mathrm{~cm}^{-3}$, depending on the size distribution of dust formed in each SN. 
Table 1. Metallicities, $[\mathrm{Fe} / \mathrm{H}]$, and abundances of $\mathrm{C}, \mathrm{O}, \mathrm{Mg}$, and $\mathrm{Si}$ relative to $\mathrm{Fe}$ in the dense shell of primordial SN II remnants for various ambient gas densities.

\begin{tabular}{|c|c|c|c|c|c|c|}
\hline$M_{\mathrm{pr}}\left(M_{\odot}\right)$ & $\log \left(Z / Z_{\odot}\right)$ & {$[\mathrm{Fe} / \mathrm{H}]$} & {$[\mathrm{C} / \mathrm{Fe}]$} & {$[\mathrm{O} / \mathrm{Fe}]$} & {$[\mathrm{Mg} / \mathrm{Fe}]$} & {$[\mathrm{Si} / \mathrm{Fe}]$} \\
\hline \multicolumn{7}{|c|}{$n_{\mathrm{H}, 0}=0.1 \mathrm{~cm}^{-3}$} \\
\hline 13 & -5.89 & -6.43 & -0.274 & -0.699 & -0.230 & 1.92 \\
\hline 20 & -5.44 & -5.20 & 0.117 & -0.595 & 0.034 & 0.410 \\
\hline 25 & -5.55 & -5.90 & 1.11 & -1.42 & -0.500 & -0.552 \\
\hline 30 & -5.33 & -5.56 & 0.566 & -0.043 & 0.739 & 0.866 \\
\hline \multicolumn{7}{|c|}{$n_{\mathrm{H}, 0}=1 \mathrm{~cm}^{-3}$} \\
\hline 13 & -4.72 & -5.15 & 1.11 & -0.555 & -0.459 & 1.01 \\
\hline 20 & -4.68 & -5.53 & 0.992 & 0.585 & 1.16 & 1.87 \\
\hline 25 & -4.79 & -5.23 & 1.09 & -0.412 & 0.407 & 0.989 \\
\hline 30 & -4.60 & -5.11 & 0.797 & 0.242 & 1.09 & 1.26 \\
\hline \multicolumn{7}{|c|}{$n_{\mathrm{H}, 0}=10 \mathrm{~cm}^{-3}$} \\
\hline 13 & -4.40 & -4.13 & 0.284 & -2.54 & -3.89 & 0.599 \\
\hline 20 & -4.09 & -4.92 & 0.946 & -2.15 & -1.80 & 2.14 \\
\hline 25 & -3.91 & -5.10 & 1.60 & 0.122 & 0.232 & 2.34 \\
\hline 30 & -3.84 & -5.11 & -0.207 & 0.375 & -1.23 & 2.66 \\
\hline
\end{tabular}

\section{Metallicities and Elemental Abundances of Population II.5 Stars}

In this section we discuss the influence of dust on the elemental composition of Population II.5 stars that are expected to form in the dense shell of Population III SNRs (Mackey et al. 2003; Salvaterra et al. 2004; Machida et al. 2005). As shown in the last section, the dust grains surviving the destruction but not injected into the ISM are piled up in the dense SN shell after $10^{5}-10^{6} \mathrm{yr}$. This implies that the elemental composition of these piled-up grains can play an important role in the elemental abundances of Population II.5 stars. Furthermore, the existence of dust in the shell may enable the formation of stars with solar mass scales through its thermal emission if the gas is enriched to the critical metallicities (Omukai et al. 2005; Schneider et al. 2006; Tsuribe \& Omukai 2006). Thus, we calculate the metallicities and metal abundances in the dense shell based on the elemental composition of piled-up grains, and compare with the observed abundance patterns of low-mass hyper-metal-poor (HMP) and ultra-metal-poor (UMP) stars.

Table 1 summarizes the calculated metallicities and elemental abundances in the dense shell of SN II remnants as a function of the ambient gas density. It should be noted that the metallicity in the shell ranges from $10^{-6}$ to $10^{-4} Z_{\odot}$, which is considered to cause the formation of stars with $0.1-1 M_{\odot}$. In addition, most of the calculated $[\mathrm{Fe} / \mathrm{H}]$ abundances are in the range of -6 to -4.5 , which is in good agreement with those for HMP and UMP stars. We also plot in Figure $2 \mathrm{a}$ the abundances of $\mathrm{C}, \mathrm{O}, \mathrm{Mg}$, and Si relative to Fe in the shell for $n_{\mathrm{H}, 0}=0.1$ and $1 \mathrm{~cm}^{-3}$. We can see that the calculated abundances of $\mathrm{Mg}$ and Si showing 1-100 times overabundances are consistent with the observations of HMP and UMP stars. Because the elemental composition of dust piled up in the shell can reproduce the abundance patterns of $\mathrm{Fe}, \mathrm{Mg}$, and $\mathrm{Si}$ in HMP and UMP stars, it is considered that the transport of dust separated from metal-rich gas within SNRs can be attributed to the elemental compositions of HMP and UMP stars, if they are Population II.5 stars.

However, as can be seen from Figure $2 a$, no model considered here can reproduce $10^{2}-10^{4}$ times excesses of $\mathrm{C}$ and $\mathrm{O}$ observed in HMP stars. One of the reasons is that 

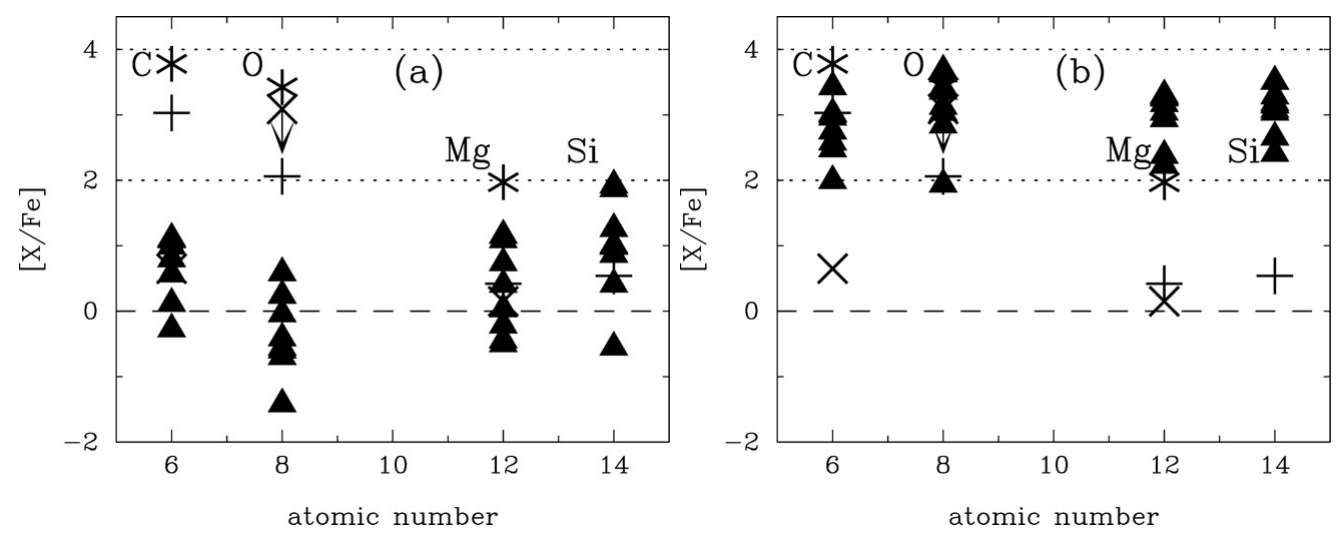

Figure 2. Abundances of C, O, Mg and Si relative to Fe in the dense shell of SN II remnants for $n_{\mathrm{H}, 0}=0.1$ and $1 \mathrm{~cm}^{-3}$ (filled triangles); $(a)$ derived from the elemental composition of the grains piled up in the shell, and $(b)$ derived from the elemental composition of the piled-up grains and the gas outside the innermost Fe layer. For observational data of HMP and UMP stars, the 3-D corrected abundances are adopted and are denoted by plus (HE0107-5240 with $[\mathrm{Fe} / \mathrm{H}]=-5.62$, Collet et al. 2006), asterisk (HE1327-2326 with $[\mathrm{Fe} / \mathrm{H}]=-5.96$, Frebel et al. 2008), and cross (HE0557-4840 with $[\mathrm{Fe} / \mathrm{H}]=-4.75$, Norris et al. 2007$)$.

in the calculation we assumed that the metal-rich gas in the SN ejecta does not mix with the gas in the shell. Then we examine the abundance patterns in the shell by assuming that besides the piled-up grains, the gas outside the innermost Fe layer in the ejecta is incorporated into the shell. The results are shown in Figure $2 b$. In this case we can reproduce the very large overabundances of $\mathrm{C}$ and $\mathrm{O}$, but the excesses of $\mathrm{Mg}$ and $\mathrm{Si}$ are too large $(\geqslant 100$ times) to agree with the observations. However, it could be possible to reproduce the abundance patterns of refractory elements observed in HMP stars unless the Si-Mg-rich layer is mixed into the shell. Unfortunately, it is still being debated to what extent the gas in the ejecta can mix into the SN shell when Population II.5 stars form. Nevertheless, we can conclude that newly formed dust in a Population III SN can have great impact on the stellar mass and metal abundance of Population II.5 stars, if the metal-rich gas is not significantly incorporated into the dense gas shell.

\section{Acknowledgements}

This work has been supported in part by a Grant-in-Aid for Scientific Research from the Japan Society for the Promotion of Sciences (18104003, 19740094).

\section{References}

Bianchi, S. \& Schneider, R. 2007, MNRAS, 378, 973

Cazaux, S. \& Spaans, M. 2004, ApJ, 611, 40

Collet, R., Asplund, M., \& Trampedach, R. 2006, ApJ, 644, L121

Frebel, A., et al., 2008, ApJ, accepted [arXiv:0805.3341]

Machida, M. N., et al., 2005, ApJ, 622, 39

Mackey, J., Bromm, V., \& Hernquist, L. 2003, ApJ, 586, 1

Norris, J. E., et al., 2007, ApJ, 670, 774

Nozawa, T., et al., 2003, ApJ, 598, 785 
Nozawa, T., et al., 2007, ApJ, 666, 955

Omukai, K., et al., 2005, ApJ, 626, 627

Salvaterra, R., Ferrara, A., \& Schneider, R. 2004, New A, 10, 113

Schneider, R., Ferrara, A., \& Salvaterra, R. 2004, MNRAS, 351, 1379

Schneider, R., et al., 2006, MNRAS, 369, 1437

Todini, P. \& Ferrara, A. 2001, MNRAS, 325, 726

Tsuribe, T. \& Omukai, K. 2006, ApJ, 642, L61 